\title{
Planar Droplet Sizing: Application to a spray of Jet A-1 kerosene
}

\author{
P. Doublet $\star^{1,2}$, C. Lempereur ${ }^{2}$, V. Bodoc ${ }^{2}$, M. Orain ${ }^{2}$, P. Gajan ${ }^{2}$ \\ ${ }^{1}$ Safran Aircraft Engines, France \\ ${ }^{2}$ ONERA The French Aerospace Lab, France \\ *Corresponding author: pierre.doublet@onera.fr
}

\begin{abstract}
Optical techniques are widely employed for their non-intrusive behavior and are applied to two-phase flow investigations. Until now, the most commonly used technique to determine the droplet size is the Phase Doppler Anemogranulometry, although it is time consuming for an overall injector characterization. An imaging technique called Planar Droplet Sizing has been used to offer an alternative and provide a spatially-resolved 2D map of the Sauter Mean Diameter (SMD). The measurement is based on the ratio between laser-induced fluorescence and scattered light intensities which are assumed to be proportional respectively to the droplet volume and droplet surface area. However, previous studies revealed that the dependence of fluorescence intensity on the droplet volume can be altered by the absorption of light in the liquid. The scattered light intensity depends on the scattering angle and intensity variations within the field of view must be avoided.

The aim of this study is to make the PDS technique operational for a Jet A-1 kerosene spray. A strong absorption of liquid kerosene appears under UV excitation at $266 \mathrm{~nm}$ making the technique unsuitable. Under visible excitation at $532 \mathrm{~nm}$, a fluorescent tracer (Pyrromethene 597) must be added to the kerosene to enhance the fluorescence signal. To prevent scattered light intensity variations within the field of view, an optimal scattering angle close to $115^{\circ}$ is required. An image processing algorithm is proposed in order to reduce the effects of multiple scattering.
\end{abstract}

\section{Keywords}

Planar Droplet Sizing, kerosene, spray, Sauter Mean Diameter

\section{Introduction}

Context

Sprays are used in many industries where, depending on the application, there are particular demands on the atomization of the liquid. In aeronautical gas turbines, the injection of liquid fuel into the highly turbulent gas flow of a combustion chamber gives rise to many complex physical phenomena: the liquid stream is stretched into ligaments and atomized into droplets that are then transported further downstream, while evaporating and enabling the combustion process. Fine atomization is required to promote vaporization and combustion. In this context, knowledge of the particle size and velocity distribution of both phases is a key factor for the design of an injection system.

The application of CFD codes to such unsteady and dense sprays is still challenging; if numerical parameters need to be tuned to fit experimental results, these data must in turn be reliable. In this respect, experiments yield essential information for the development and evaluation of atomization and spray flow models.

In order to fulfill the accuracy requirement, laser-based optical diagnoses are in steady progress. These techniques are preferred to physical probes because they do not disturb the spray and can be applied in a harsh environment where a physical probe would not survive. Although a classical pointwise technique, Phase Doppler Anemogranulometry, has been widely investigated over the last decades, whole-field measurement techniques are a promising means to reduce the test duration and cost.

\section{Planar Droplet Sizing (PDS)}

The Planar Droplet Sizing method also called LIF/Mie firstly appeared in 1993 for the characterization of a transient fuel spray [1]. This technique consists in illuminating a spray with a laser sheet. Contrary to the classical techniques as PDA (Phase Doppler Anemogranulometry) or ILIDS (Interferometric Laser Imaging for Droplet Sizing), PDS does not yield the individual particle size, but a statistical value at each point, namely the SMD, which is widely used to characterize injection devices. The pros and cons of PDS and traditional techniques are summarized in the table 1 . This is an important quantity for mass transfer processes: a small SMD indicates fine evaporation, weak penetration, efficient mixture and combustion processes. This technique is based on Mie 
scattering and fluorescence emission from droplets. The fluorescence and scattered light intensities $I_{F}$ and $I_{M}$ are assumed to be proportional respectively to the volume and the surface of the illuminated droplet [1]. A ratio of both images is therefore proportional to the SMD, also called $d_{32}$, of the size distribution of the $\mathrm{N}$ measured droplets. Thanks to this technique, a two-dimensional map of $d_{32}$ can be obtain in a few minutes.

$$
\frac{I_{F}}{I_{M}}=\frac{k_{F} \sum_{i}^{N} n_{i} d_{i}^{3}}{k_{M} \sum_{i}^{N} n_{i} d_{i}^{2}}=k \cdot d_{32}
$$

where $k_{F}, k_{M}$ and $k$ are coefficients determined experimentally. A numerical and experimental study allowed Domann and Hardalupas to analyze the accuracy of this technique [2]. More recently, Lempereur and al. [3] followed this approach in order to characterize a real aeronautical engine spray under atmospheric conditions. This work showed that the scattering angle (Figure 1.c), the droplet size and the refractive index modify the proportionality between the scattering intensity and the droplet surface. In parallel, the influence of the concentration of fluorescent dye on the proportionality between the fluorescence intensity and the droplet volume [4] was demonstrated. Optimum values of the scattering angle and the fluorescent dye concentration were obtained to improve the sizing measurement accuracy $[5,6]$ and recent studies compared the PDS measurements with the PDA ones $[3,7]$. Nevertheless in that study, ethanol was used as a surrogate fluid.

Table 1. Pros and cons of main technique used for measuring droplet diameters.

\begin{tabular}{|c|c|c|c|c|}
\hline \multirow[b]{2}{*}{ Techniqu } & Laser diffraction & PDA $[8,9]$ & ILIDS [10] & PDS [3] \\
\hline & & & & \\
\hline Pros & $\begin{array}{l}\text { - Convenient to use } \\
\text { - Large diameter } \\
\text { range }(0,1-900 \mu \mathrm{m})\end{array}$ & $\begin{array}{l}\text { - Particule } \\
\text { concentration }>100 \mathrm{p} / \\
\mathrm{mm}^{3} \\
\text { - Large diameter range } \\
(0,5-1000 \mu \mathrm{m}) \\
\text { - Simultaneous } \\
\text { measurement of } \\
\text { velocity and diameter }\end{array}$ & $\begin{array}{l}\text { - 2D measurement } \\
\left(10 \times 10 \mathrm{~mm}^{2}\right)\end{array}$ & $\begin{array}{l}\text { - 2D measurement } \\
\left(100 \times 100 \mathrm{~mm}^{2}\right) \\
\text { - Fast } \\
\text { measurement } \\
\text { - High particule } \\
\text { concentration } \\
\text { (limited by multiple } \\
\text { scattering) }\end{array}$ \\
\hline Cons & $\begin{array}{l}\text { - Integrated } \\
\text { measurement }\end{array}$ & $\begin{array}{l}\text { - Point measurement } \\
\text { - Time consuming }\end{array}$ & $\begin{array}{l}\text { - Particule } \\
\text { concentration < } \\
10 \mathrm{p} / \mathrm{mm}^{3} \\
\text { - Limited diameter } \\
\text { range }(20-1000 \mu \mathrm{m})\end{array}$ & $\begin{array}{l}\text { - Calibration with } \\
\text { PDA required }\end{array}$ \\
\hline
\end{tabular}

Focus of the study

The aim of this paper is to extend the Planar Droplet Sizing technique to a spray of Jet A-1. The first part of this article highlights a review of the implementation of this technique on a swirled spray using ethanol and Rhodamine $6 G[3,6]$. The extension to the Jet A-1 requires knowledge of its optical properties in order to accurately define the experimental setup. These issues will be discussed in the second part. The PDS technique implementation and first results will be the topic of the last part.

\section{PDS implementation on an aeronautical swirled spray}

Recent investigations at ONERA focused on the implementation of this technique to characterize the spatial droplet size distribution in the spray of an aeronautical swirled injection system used in actual combustion chambers [3, 6]. Tangential air intakes create a swirl around the injection axis in order to promote flame stabilization and flow mixture. Experiments are conducted at ambient temperature on the unsteady spray generated downstream of the injector. The liquid used for the simulation is ethanol, because its surface tension is close to that of kerosene $\left(\sigma_{e t h}=22 \mathrm{mN} / \mathrm{m}\right.$ and $\left.\sigma_{k e r}=23,3 \mathrm{mN} / \mathrm{m}\right)$. Since ethanol is not naturally fluorescent, it was doped with a dye. Rhodamine $6 \mathrm{G}$ was chosen because of its optimal excitation wavelength (529 $\mathrm{nm})$ and its 
high quantum yield (0.95) in these conditions. An intensified camera was used with filters for fluorescence and scattered light images. The study focuses on the parameters which alter respectively fluorescence and scattered light as the particle number concentration, the scattering angle or the dye concentration. The main parameter that affects the relation between fluorescence intensity and particle volume is the dye concentration c. The relation can be rewritten as follows:

$$
I_{F}=k_{F} \sum n_{i} d_{i}^{F(c)}
$$

On one hand, fluorescence intensity is generally low and the dye concentration must be large enough in order to yield a sufficient signal. On the other hand, the light absorption increases with the dye concentration. A concentration of Rhodamine $6 \mathrm{G}$ of $1 \mathrm{mg} / \mathrm{L}$ was sufficient to give an exploitable fluorescent signal on the intensified camera. [4].

The droplet size and the scattering angle $\alpha$ alter the relation between the scattered light intensity and the droplet surface area. The relation can be expressed as follows:

$$
I_{S}=k_{S} \sum n_{i} d_{i}^{M\left(d_{i}, \alpha\right)}
$$

Investigations on mean scattered light images enabled to determine the parameters that affect the measurement accuracy. As can be seen in Figure 1, contrary to the fluorescence image, the scattering image was not symmetric on both sides of the nozzle axis and an attenuation of more than $20 \%$ between the amplitudes of the two peaks $A$ and $B$ can be measured. This attenuation was due to the variation of the scattering angle within the field of view (scattering angle centered at $90^{\circ}$ ). To limit this effect, two corrections were proposed. First, the angle of view was reduced by increasing the focal length and observation distance. Then, a favourable mean scattering angle was determined (close to $100^{\circ}$ ) to lessen the intensity variation within the field of view. In this angular range, the exponent $\mathrm{M}$ only depends on the droplet diameter. A theoretical approach enables to estimate the relation between the scattered intensity and the surface area. In the range of interest $(1-100 \mu m)$, the $d^{2}$ law is invalidated: the $\mathrm{M}$ exponent steadily increases from 1 to 2 with the diameter. The best power law fit for diameters between 5 and $100 \mu \mathrm{m}$ is obtained for $M=1,7$.
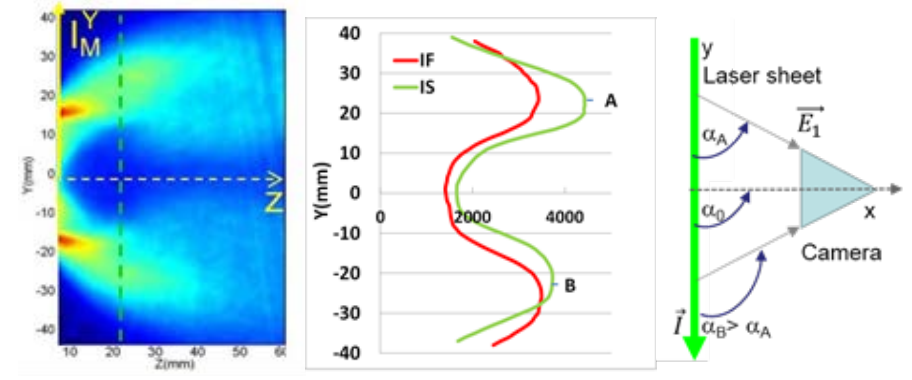

Figure 1. (a) Mean Mie scattered light image (b) vertical Y-profiles on Mie and LIF images at $Z=20 \mathrm{~mm}$ from the injection point (c) definition of the local scattering angle for $\alpha_{0}=90^{\circ}$.

PDS and PDA measurements were compared in order to validate the PDS results. The knowledge of exponents $M$ and $F$ helped understanding the differences between both techniques: the diameter $d_{F M}$ calculated with the PDS technique has the unit of a diameter at a power roughly equal to 1,3 for $F=3$ and $M=1,7$.

$$
\left(d_{F M}\right)^{F-M}=\frac{\sum d^{F}}{\sum d^{M}}
$$

Since the PDS ratio was not found to be proportional to $d_{32}$, a calibration is required for the whole diameter range. The method consists in creating a correspondence between values of Sauter Mean Diameter measured with PDA and ratios obtained by PDS image processing at the same points. A power law fit was obtained and can be used to build a Look Up Table (LUT) to convert PDS images into $d_{32}$ maps. This calibration has been validated on several diameters beyond a distance of $Z=20 \mathrm{~mm}$ from the injection point. Closer to the injection, some discrepancies occurred between PDS and PDA measurements due to the presence of large, non-spherical liquid structures. Moreover, a higher density of particles may induce multiple scattering. As a result, none of those techniques is reliable close to the injector where the density of particles becomes high. 
For more applied studies involving on the influence of fluid properties variation over the atomisation process, it is necessary to transpose these results obtained with ethanol to the Jet A-1.

\section{PDS extension to Jet A-1}

Characterization of jet A-1 optical properties

Before implementing the PDS technique on a Jet A-1 spray, some investigations of its optical properties have to be carried out in order to define precisely the experimental setup (excitation wavelength, camera orientation, ...) and to estimate the exponents $\mathrm{M}$ and $\mathrm{F}$. The idea is to quantify the absorption coefficient $\mathrm{k}$ and to insure that the kerosene fluorescence is high enough to obtain reliable fluorescence images.

The first attempt consisted in exciting in the UV (at $266 \mathrm{~nm}$ ) because of the well-known fluorescence of the aromatics contained in kerosene vapours [11]. In order to extend these results to liquid kerosene, experiments are realized with a 10mm-side cubic quartz test cell containing liquid kerosene. As shown in Figure 2.a, a spectrometer and an intensified camera are placed on opposite sides of the cell to enable simultaneous fluorescence and absorption measurements. The laser is a Quantel Brilliant one, the spectrometer is a HR2000+ from Ocean Optics and the intensified camera is a Princeton PIMAX II.

The normalized fluorescence spectrum of liquid kerosene, plotted in Figure 2.b, exhibits the same shape as the vapour spectrum one obtained in previous studies [11]. The first fluorescent band is due to the mono-aromatic molecules whereas the di-aromatics ones are responsible for the second band [11]. However, a shift of the emission spectrum of liquid kerosene to higher wavelengths is observed, probably due to the differences in energy levels between liquid and vapour phases.

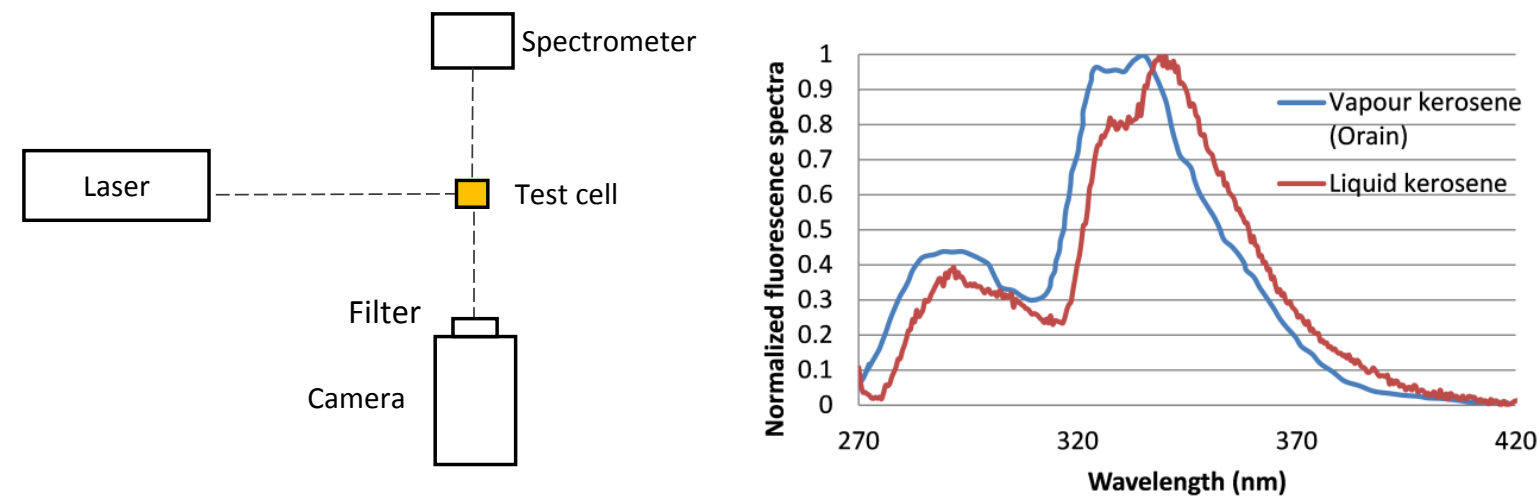

Figure 2. (a) Experimental set-up for the fluorescent and absorption measurements (b) Normalized fluorescence spectra of liquid and vapour Jet A1.

The images point out a strong absorption of the liquid kerosene at this excitation wavelength: laser beam extinction appeared a few millimetres downstream from the entrance window. In order to quantify this absorption, the parameter of interest is the extinction coefficient $\mathrm{k}$ which is the imaginary complex part of the refractive index. This parameter is involved in the Beer-Lambert law:

$$
I=I_{0} \exp \left(-\frac{4 \pi k}{\lambda} x\right)
$$

where $I_{0}$ is the laser intensity at the entrance window of the test cell, $x$ the optical path in the medium and $I$ is the laser intensity at the probe volume. The factor $\mathrm{k}$ is obtained from the processing of the images of the laser beam propagation in the cell. For a $266 \mathrm{~nm}$ excitation wavelength, the value of the extinction coefficient $k$ is high, close to $1,3 \cdot 10^{-2}$. The exponent $\mathrm{F}$ involved in the relation between fluorescence intensity and droplet volume can be estimated thanks to simulations of internal laser field excitation [12]. It is not equal to 3 but close to 2: as a result, the fluorescence intensity is not proportional to the volume of the droplet but rather to its surface area. Therefore, the PDS technique using this excitation wavelength seems to be unsuitable because the ratio of fluorescence to scattered light intensities is not proportional to the Sauter Mean Diameter but is roughly constant. To solve this problem, the excitation wavelength is changed from $266 \mathrm{~nm}$ to $532 \mathrm{~nm}$. At this wavelength, a low absorption is measured $\left(k=7,9.10^{-9}\right)$ and the coefficient $\mathrm{F}$ is much closer to $3(F=2,91)$. Nevertheless, considering an excitation wavelength of $532 \mathrm{~nm}$, the fluorescence signal is very low and will be a limiting factor. The use of a fluorescent dye is thus required as in the ethanol case. Rhodamine 6G was used with ethanol but is not miscible with kerosene; Pyrromethene 597-8C9 is selected: its wavelength absorption peak is centered on $525 \mathrm{~nm}$ corresponding to a fluorescent emission between $540 \mathrm{~nm}$ and $700 \mathrm{~nm}$. Several concentrations are tested in order 
to determine the best compromise: a concentration of $1 \mathrm{mg} / \mathrm{L}$ leads to an extinction coefficient of $3.10^{-7}$ and a coefficient $F$ of 2,90 . Table 2 summarizes the values of the absorption coefficient and coefficient $F$ according to the mixture types and excitation wavelengths.

Table 2. Extinction and $F$ coefficients for the different mixtures and excitation wavelengths.

\begin{tabular}{c|ccc}
\hline \hline Mixture & Excitation wavelength $(\mathrm{nm})$ & $\begin{array}{c}\text { Extinction } \\
\text { coefficient k }\end{array}$ & Coefficient F \\
\hline \hline Kerosene & 266 & $1,3.10^{-2}$ & 1,99 \\
Kerosene & 532 & $7,9.10^{-9}$ & 2,91 \\
Kerosene + Pyrromethene $(1 \mathrm{mg} / \mathrm{L})$ & 532 & $3.10^{-7}$ & 2,90
\end{tabular}

\section{PDS implementation and measurements}

Experimental set-up

A hollow-cone Jet A-1-spray, generated from a Delavan nozzle, is investigated at ambient temperature, atmospheric pressure and with a liquid injection pressure set to 5 bars which corresponds to a liquid flow rate of $8 \mathrm{~L} / \mathrm{h}$. In this case, the properties of liquid kerosene are the following: $\sigma=23,30 \mathrm{mN} / \mathrm{m}, \rho=798,78 \mathrm{~kg} / \mathrm{m}^{-3}$ and $v=1.6405 .10^{-6} \mathrm{~m}^{2} / \mathrm{s}$. In the present study, visualisations in longitudinal sections of the spray are considered. As shown in Figure 3, the spray core is illuminated at $532 \mathrm{~nm}$ with a Quantel Twins BSL Nd:Yag laser. A Princeton PIMAX IV camera with a GenIII intensifier is chosen for these experiments. The quantum efficiency (QE) of the camera is close to $50 \%$ in the dye emission spectral range $(>532 \mathrm{~nm})$. For the fluorescence signal, a high pass filter with a cut-off wavelength at $540 \mathrm{~nm}$ is used (the optical density is equal to 7 in the blocking range), whereas, for the Mie signal, a band pass filter centered at $532 \mathrm{~nm}$ (FWHM 10nm) is used (the optical density is equal to 6 in the blocking range). The optical settings are facilitated thanks to sequential acquisitions. Both LIF and Mie signals come from the same camera and viewing point, which suppresses potential discrepancies originating from the sensors, the dewarping or from different optical paths. Moreover, to ensure reproducible measurements, the pressurized tank is equipped with an expansion valve which limits the pressure fluctuations under 0,01 bar. The dynamic range of the camera is, for the LIF signal, a critical parameter to visualize the largest droplets without saturation while enabling the detection of the smallest ones, keeping in mind that the intensity is proportional to the volume of the particle. The best figures are obtained when operating the camera at the lowest pixel readout rate, in order to generate the lowest readout noise. Thus, the frame rate is kept at its minimum, namely 5 images/s in these experiments. The LIF and Mie averaged images should be obtained from several thousands of images to ensure a good statistical convergence. The top of the fluorescence and scattered images corresponds to the nozzle exit. In order to prevent saturations the spray formation region is not illuminated by the laser sheet. All of the images are dewarped in the measurement plane and presented in millimeters.

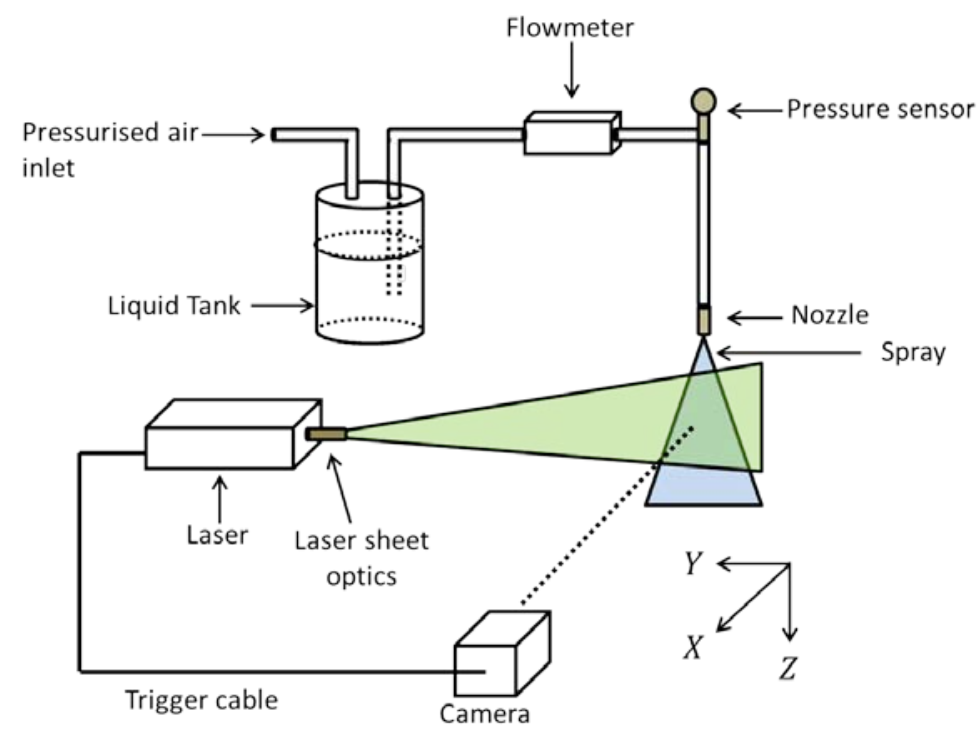

Figure 3. Experimental set-up for the LIF/MIE technique implementation. 


\section{Scattered light images}

Since the variation of the scattering angle within the field of view may be responsible for strong intensity variations, it is interesting to look for an optimal configuration where this fluctuation is minimized. The theoretical evolution of the scattered light intensity versus $\alpha$ is calculated for $\alpha$ ranging from $60^{\circ}$ to $120^{\circ}$ and for various droplet diameter distributions. As shown in Figure 4, the slope of $I(\alpha)$ is important in forward scattering and tends towards zero in the $110-120^{\circ}$ range. For this PDS implementation on kerosene, a mean scattering angle close to $115^{\circ}$ is chosen. To study the effect of the scattering angle on the scattered light intensity, two critical values are considered: the camera is placed at $70^{\circ}$ and $115^{\circ}$. With a $105 \mathrm{~mm}$ focal length and a $13 \mathrm{~mm} \times 13 \mathrm{~mm} \mathrm{CCD}$ sensor, an angle of view of $\pm 2,5^{\circ}$ is achieved. At a distance of $840 \mathrm{~mm}$, a $90 \mathrm{~mm}$-side field can be visualized.

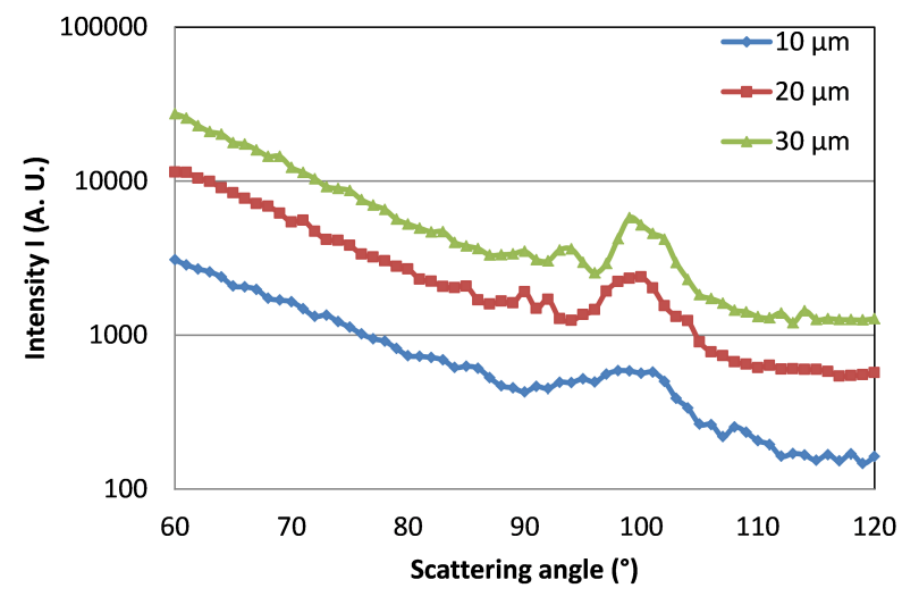

Figure 4. Variation of the scattered light intensity versus $\alpha$ for several log-normal droplet distributions (radius mean values equal to $10,20,30 \mu \mathrm{m}$ and standard deviation equal to $10 \%$ ). The calculations have been computed with an laser excitation wavelength of $532 \mu \mathrm{m}$ and a refractive index of 1,45.

As can be seen on the averaged images (Figure 5), for a scattering angle of $70^{\circ}$, a strong dissymmetry appears on the Mie image only. In each case $\left(70^{\circ}\right.$ and $\left.115^{\circ}\right)$, the laser sheet is coming from the left side. The left side of this image exhibits higher levels than the right one due to the angular sensitivity described in Figure 4 . At a scattering angle of $115^{\circ}$, the scattered image is roughly symmetrical about the injection axis. The choice of this angle results from a theoretical study to limit the angular effect. As expected, the fluorescence signal does not depend on the scattering angle because fluorescence is supposed to be an isotropic phenomenon. For the following experiments and investigations, a scattering angle of $115^{\circ}$ is chosen.

Scattering angle

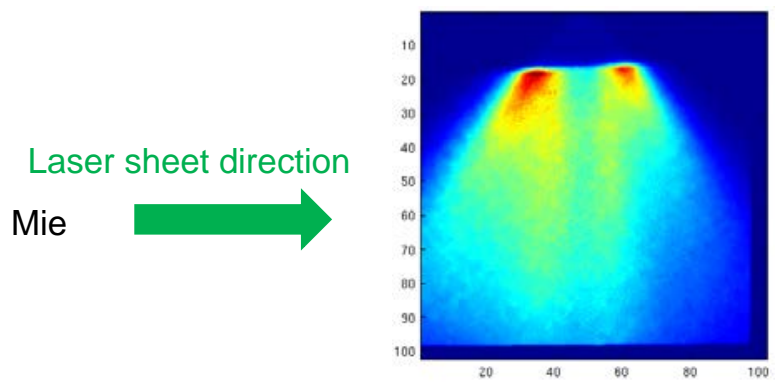

LIF

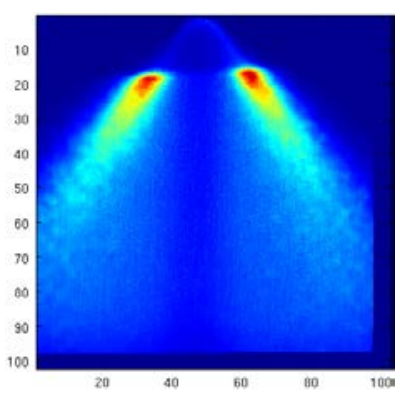

$115^{\circ}$
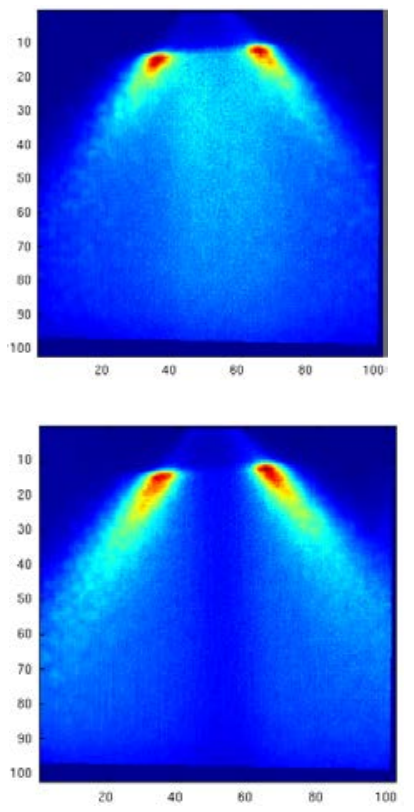

Figure 5. LIF/Mie averaged images for $\alpha=70^{\circ}$ and $115^{\circ}$. 
In both configurations, the intensity level of the Mie images seems overestimated inside the spray. Signal is detected where no droplets are present because the light scattered by a droplet illuminated by the laser may in turn be scattered by droplets that are not contained in the laser sheet. This multiple scattering effect is here mainly visible on the Mie images. Actually, when the density of droplets becomes high, a method based on spatial intensity modulation of the laser sheet, SLIPI [13] has proved effective to differentiate between the contributions arising from direct and multiple scattered light. The approach enables the removal of undesired diffuse light coming from the spray itself or from reflections in the background. This technique will be implemented in a near future on this spray.

Meanwhile, in order to reduce the contribution of multiple scattering in the signal, an image processing algorithm based on top hat filtering is applied to the images. The idea consists in filtering out the low frequency image information which corresponds to the surrounding background (from multiple scattering) while preserving the high frequencies. Since the spray is not too dense, individual droplets are visible in each image and can be extracted by this procedure which removes the contribution of the local background as illustrated in Figure 6 . The result is still noisy here and should be obtained from a longer sequence of images - at least a few thousand - in order to achieve a better description of the spray. The idea of high pass filtering in spray images was firstly shown by [14]. Some precautions have to be taken because this approach can also remove some relevant information. Anyhow, this method seems promising and requires further investigation and comparison with the SLIPI solution.
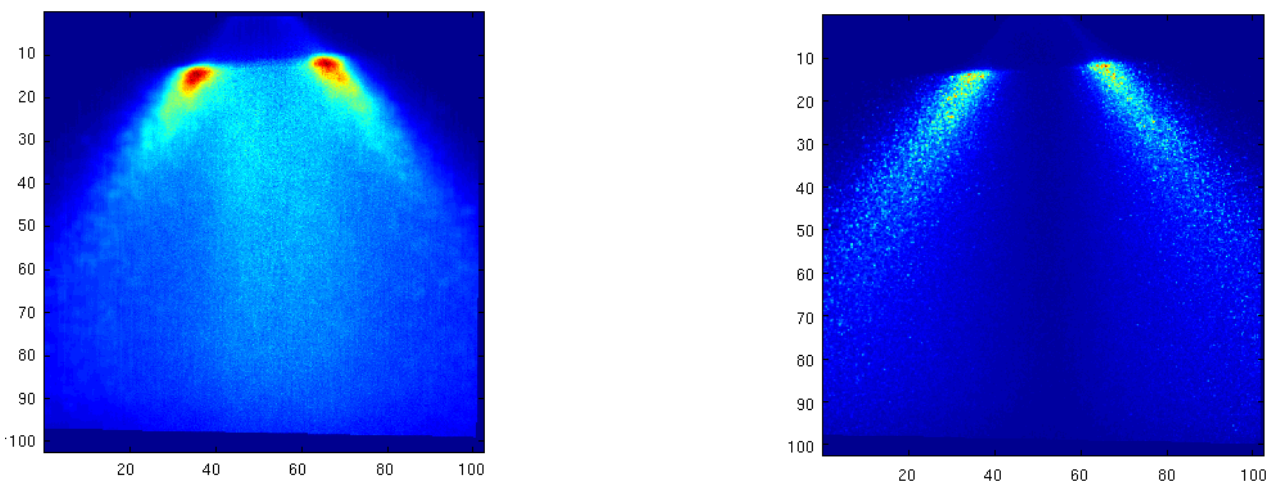

Figure 6. (a) Averaged scattered image (b) Averaged image from top hat filtered images of the sequence.

\section{Sauter Mean Diameter mapping}

The LIF/Mie ratio calculated from averaged images is presented in Figure 7. Although the Mie image needs to be improved, the result is qualitatively satisfactory: the map is roughly symmetrical about the injection axis, the minimum value of the ratio obtained in the spray core is three to four times smaller than the one at the edges, which is consistent with the SMD measured by PDA. Complementary PDA measurements will enable to achieve absolute PDS mapping.
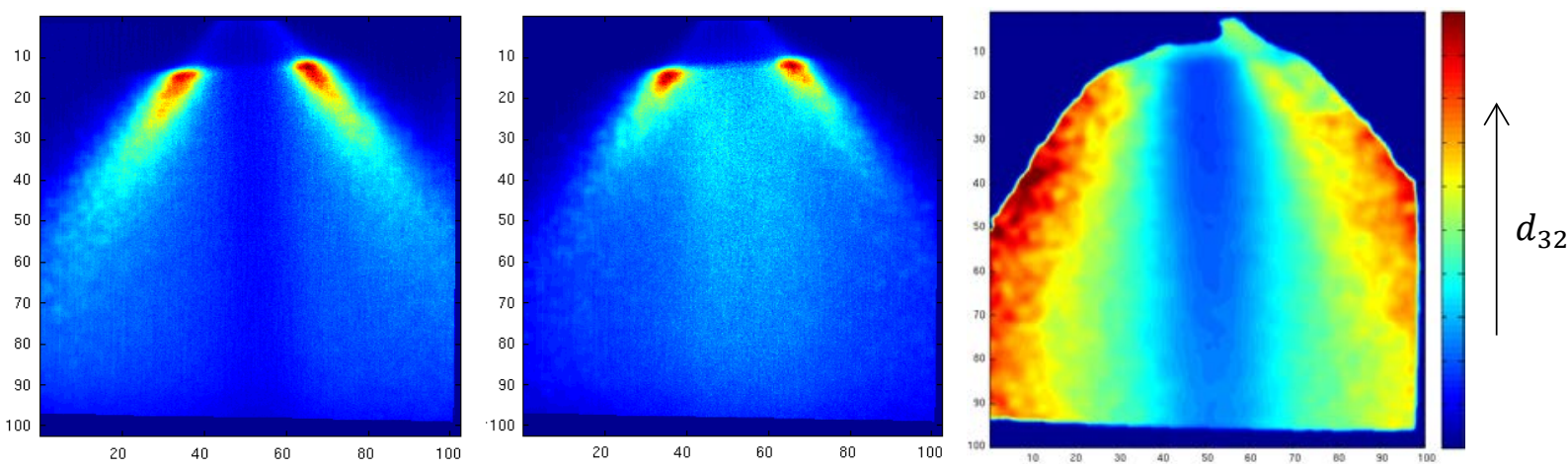

Figure 7. LIF and MIE images and their ratio proportional to the droplet SMD. 


\section{Conclusions and perspectives}

Planar Droplet Sizing is an imaging technique providing a spatially-resolved 2D map of the Sauter Mean Diameter in a few minutes contrary to point measurement techniques. A preliminary study enabled to adapt it to an ethanol spray by investigating the influence of optical and geometrical parameters on the measurement accuracy. The present investigation highlights its extension to Jet A-1 spray. The optical properties of liquid kerosene result in a strong absorption under UV excitation which makes the PDS technique unsuitable. The use of fluorescent dye appears necessary in order to increase the fluorescence signal of liquid kerosene under visible excitation at 532 $\mathrm{nm}$. The effect of the scattering angle is also investigated with theoretical and experimental approaches. A scattering angle close to $115^{\circ}$ seems to be adapted to prevent scattered light intensity variation within the field of view. Under these conditions, the LIF/Mie ratio exhibits the symmetry of the spray around the injection axis and provides a relevant order of magnitude of the SMD variations within the spray. Since scattered light images are altered by multiple scattering, an image processing algorithm is proposed to reduce this effect, prior to the implementation of the SLIPI technique.

\section{Acknowledgements}

This work is conducted in cooperation between ONERA and SAFRAN AIRCRAFT ENGINES (Safran Group). The authors gratefully acknowledge the ANRT (Association Nationale de la Recherche et de la Technologie) which supports part of the PhD.

\section{Nomenclature \\ $\sigma_{\text {eth }}$ Ethanol surface tension $\left[\mathrm{kg} / \mathrm{s}^{2}\right]$. \\ $\sigma$ Kerosene surface tension $\left[\mathrm{kg} / \mathrm{s}^{2}\right]$. \\ $\rho$ Kerosene density $\left[\mathrm{kg} / \mathrm{m}^{3}\right]$. \\ $v$ Kerosene kinematic viscosity $\left[\mathrm{m}^{2} / \mathrm{s}\right]$.}

\section{References}

[1] Kamimoto, T., Yokota, H., and Kobayashi, H., 1989, SAE Transactions Journal of Engines, 98, paper\#890316.

[2] Domann, R., Hardalupas, Y., 2001, Particle \& Particle Systems Characterization, 18, pp. 3-11.

[3] Lempereur, C., Bodoc, V., Brettar, J. and Gajan, P., Sep. 2016, 15 ${ }^{\text {th }}$ CFTL.

[4] Charalampous, G. and Hardalupas, Y., 2011, Applied Optics, 50 (9), pp.1197-1209.

[5] Charalampous, G. and Hardalupas, Y., 2011, Applied Optics, 50 (20), pp. 3622-3637.

[6] Brettar, J., Thesis, 2015, ONERA

[7] Mishra, Y., Kristensson, E. and Berrocal, E., 2014, Optics Express, 22 (4), pp. 4480-4492.

[8] Sankar, S. V., Inenaga, A. S. and Bachalo, W. D., July 20.-23. 1992, $6^{\text {th }}$ International Symposium on Applications of Laser Anemometry to Fluid Mechanics.

[9] Kawahara, N., Tomita, E. and Okano, H., 2012, ICLASS.

[10] Hardalupas, Y., Sahu, S., Taylor, A. MKP and Zarogoulidis, K., 2010, Experiments in fluids, 49 (2), pp. 417434.

[11] Orain, M., Baranger, P., Ledier, C., Apeloig, J. and Grisch, F., 2014, Applied Physics B, 116 (3), pp. 729-745.

[12] Mees, L., Grehan, G. and Gouesbet, 2001, Optics Communications, 194, pp. 59-65.

[13] Berrocal, E., Kristensson, E., Hottenbach, P., Grünefeld, G. and Aldén M., May 15. - 18. 2011, 23rd Annual Conference on Liquid Atomization and Spray Systems.

[14] Smallwood, G. J, Guelder, O. L and Snelling, D. R,1994, $25^{\text {th }}$ Symposium on Combustion/ The Combustion Institute, pp. 371-379. 